\title{
BMJ Open Barriers and facilitators of physical activity in knee and hip osteoarthritis: a systematic review of qualitative evidence
}

\author{
Archontissa M Kanavaki, ${ }^{1,2}$ Alison Rushton, , ${ }^{1,2,3}$ Nikolaos Efstathiou, ${ }^{4,8}$ \\ Asma Alrushud, ${ }^{1,5}$ Rainer Klocke, ${ }^{6}$ Abhishek Abhishek, ${ }^{7}$ Joan L Duda ${ }^{1,2}$
}

To cite: Kanavaki AM, Rushton A, Efstathiou $\mathrm{N}$, et al. Barriers and facilitators of physical activity in knee and hip osteoarthritis: a systematic review of qualitative evidence. BMJ Open 2017;7:e017042. doi:10.1136/ bmjopen-2017-017042

- Prepublication history and additional material for this paper are available online. To view these files, please visit the journal (http://dx.doi.org/10. 1136/bmjopen-2017-017042).

Received 28 March 2017 Revised 12 July 2017 Accepted 31 July 2017

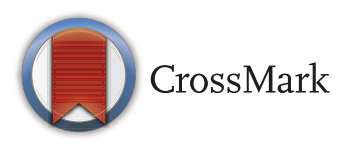

For numbered affiliations see end of article.

Correspondence to Archontissa M Kanavaki; amk377@bham.ac.uk

\section{ABSTRACT}

Physical activity (PA), including engagement in structured exercise, has a key role in the management of hip and knee osteoarthritis $(0 \mathrm{~A})$. However, maintaining a physically active lifestyle is a challenge for people with OA. PA determinants in this population need to be understood better so that they can be optimised by public health or healthcare interventions and social policy changes. Objectives The primary aim of this study is to conduct a systematic review of the existing qualitative evidence on barriers and facilitators of PA for patients with hip or knee $0 A$. Secondary objective is to explore differences in barriers and facilitators between (1) lifestyle PA and exercise and (2) PA uptake and maintenance.

Methods Medline, Embase, Web of Science, Cumulative Index to Nursing and Allied Health Literature, SPORTDiscus, Scopus, Grey literature and qualitative journals were searched. Critical Appraisal Skills Programme-Qualitative checklist and Lincoln and Guba's criteria were used for quality appraisal. Thematic synthesis was applied.

Findings Ten studies were included, seven focusing on exercise regimes, three on overall PA. The findings showed a good fit with the biopsychosocial model of health. Aiming at symptom relief and mobility, positive exercise experiences and beliefs, knowledge, a 'keep going' attitude, adjusting and prioritising PA, having healthcare professionals' and social support emerged as PA facilitators. Pain and physical limitations; non-positive PA experiences, beliefs and information; 0A-related distress; a resigned attitude; lack of motivation, behavioural regulation, professional support and negative social comparison with coexercisers were PA barriers. All themes were supported by high and medium quality studies. Paucity of data did not allow for the secondary objectives to be explored.

Conclusion Our findings reveal a complex interplay among physical, personal including psychological and social-environmental factors corresponding to the facilitation and hindrance of PA, particularly exercise, engagement. Further research on the efficacy of individualised patient education, psychological interventions or social policy change to promote exercise engagement and lifestyle PA in individuals with lower limb $O A$ is required.

Trial registration number CRD42016030024.

\section{Strengths and limitations of this study}

- This systematic review is the first to identify, appraise and synthesise the existing qualitative research on barriers and facilitators to physical activity $(\mathrm{PA})$ in knee and hip osteoarthritis.

- Rigorous methods have been applied, informed by the Centre for Reviews and Dissemination and Cochrane Qualitative Research Methods Group guidelines and reported according to the Preferred Reporting Items for Systematic Reviews and MetaAnalyses and Enhancing Transparency in Reporting the Synthesis of Qualitative Research statements.

- The majority of the included studies $(7 / 10)$ focused on exercise barriers and facilitators; therefore, barriers and facilitators of more general lifestyle PA might not be fully captured.

- Papers written in English-language only were included.

Osteoarthritis $(\mathrm{OA})$ is the the most common joint disease and main cause of disability in older adults. ${ }^{1}$ OA management focuses on analgesia and non-pharmacological modalities such as exercise and weight loss. ${ }^{2}$ Exercise, that is, structured and purposeful physical activity $(\mathrm{PA}){ }^{3}$ reduces pain and improves function in people with knee or hip OA. ${ }^{4-9}$ However, despite the positive effects on symptoms, exercise interventions do not promote sustained behaviour change. ${ }^{10} 11$ Just like exercise, PA associates with better physical function ${ }^{12-14}$ and even modest increase in PA (from sedentary to light intensity PA) improves arthritis pain. ${ }^{15}$ At the population level, it is simpler to promote PA in people with painful OA for example, via radio and television, than promoting exercise as that will require a greater behaviour change and may need continued support of trained physiotherapists. However, existing evidence suggests that people with lower limb OA have such low PA levels that they 
gain no health benefits from it. ${ }^{16-18}$ Thus, there is a need to understand the determinants of reduced PA in people with symptomatic OA so that these can be optimised to promote PA.

The disease-specific determinants of PA in those with lower limb OA, for example, symptom severity and physical function ${ }^{19-23}$ are relatively well understood, but the psychological, social and environmental determinants of PA in OA have not been adequately examined. ${ }^{21}{ }^{22}$ Understanding these factors is of great importance as pain makes PA an aversive experience leading to activity avoidance $^{24-27}$ and pain is influenced by psychological and environmental factors. ${ }^{18252829}$ A recent scoping review identified several psychological and environmental barriers and facilitators of exercise in people with hip or knee OA. ${ }^{23}$ However, scoping reviews lack the methodological rigour of systematic reviews (SRs).$^{30} \mathrm{~A}$ SR of qualitative data holds promise for a thorough and in-depth understanding of the modifiable psychosocial factors predicting PA behaviour.

The objectives of this study were to: identify, appraise and synthesise the existing qualitative evidence on barriers and facilitators to PA in hip or knee OA; explore differences in barriers and facilitators between lifestyle PA accrued in daily activities and those reported in regard to structured exercise programme specifically and between PA uptake and maintenance.

\section{METHODS}

This SR was registered with the International Prospective Register of SRs (CRD42016030024) and its protocol reported previously. ${ }^{31}$ The reporting follows the Preferred Reporting Items for SRs and Meta-Analyses and the Enhancing Transparency in Reporting the Synthesis of Qualitative Research statements (see online supplementary file 1$)$.

\section{Population, Intervention, Comparators, Outcomes were adapted to inform eligibility \\ Population}

Study participants were adults with physician diagnosed or radiographic (Kellgren and Lawrence grade $\geq 2$ ) hip or knee OA or met classification criteria for $\mathrm{OA}$ at these joints. ${ }^{32}$ If a study included people with other arthritis, for example, rheumatoid arthritis, they were included if people with knee or hip OA were the largest proportion. Studies with participants awaiting total joint replacement were excluded.

\section{Outcomes}

The perceptions of barriers and facilitators that influence uptake or maintenance of PA were the study outcomes. Studies were included if they explored the factors/ barriers/facilitators/motivation to engagement in PA or addressed the experience of people with hip or knee OA regarding PA or exercise.
Study designs

Qualitative or mixed methods studies.

\section{Language}

Published in English.

\section{Information sources}

Medline (Ovid Medline(R) in-process and other non-indexed citations and Ovid Medline(R) 1946 to present, Ovid), Embase (1974 onwards, Ovid interface), PhychINFO (1967 onwards, OVID), Web of Science, Cumulative Index to Nursing and Allied Health Literature, SPORTDiscus and Scopus were searched up to 31 of December 2015. Grey literature sources were explored, that is, OpenGrey, National Health Service evidence. The search strategy was complemented by hand search of qualitative-research-centred journals screening of references of included articles and contacting researchers active in the field.

\section{Search}

The search strategy contained exhaustive keyword combinations for each of the four concepts of interest, that is, knee or hip OA; PA/exercise; facilitators, barriers, motivation, uptake, maintenance; qualitative studies (see online supplementary file 2).

\section{Study selection}

The search and study selection was conducted by two researchers independently (AMK and AsA). Endnote V.X7 was used for data management. Citations and abstracts were imported and duplicates removed. After title/abstract screening, full text of potentially relevant studies were assessed and additional information was sought from authors where necessary. If consensus was not reached between the two researchers, a third reviewer was consulted (AR).

\section{Data collection and appraisal}

All text under the sections of 'results' and 'findings' of the selected studies was considered as data items. Where findings and discussion were presented together, the whole section was considered for analysis. Data items were entered into and managed with NVivo V.11 qualitative data analysis software (QSR International).

Quality appraisal aimed to assess the reporting, methodological rigour and conceptual consistency of the included studies ${ }^{33}$ and to identify and discard low-quality studies. Two approaches were used, which complement each other ${ }^{31}$ : (a) the Critical Appraisal Skills ProgrammeQualitative Checklist. ${ }^{34}$ Studies were rated as high, medium and low quality if they met $\geq 8,5-7$ and 4 or fewer criteria, respectively; (b) the evaluative criteria of credibility, transferability, dependability and confirmability that assess the trustworthiness of the study. Studies were rated high, medium, and low quality if they met $\geq 3,2$, or one and less criteria. ${ }^{35}$ Two reviewers independently appraised the selected studies (AK and NE). 
The phenomenon of interest was the description and interpretation of OA patients' perceptions and experiences regarding what facilitates, motivates or hinders them from engaging in PA. In addition, observed differences in facilitators and barriers to uptake and maintenance of PA (exercise and lifestyle PA) were also included.

\section{Synthesis of results}

Data were analysed by thematic synthesis. ${ }^{36}$ First, authors' interpretations and informants' quotes were coded separately, line by line. Codes of original themes, subthemes and codes clearly referring to other types of arthritis where excluded from the synthesis. Next, descriptive themes were formed through code merging and grouping in a highly iterative process, creating a hierarchical tree. To form the analytical themes, a data-driven analysis was initially conducted to allow an inductive interpretation. A group (AMK, NE, AR, JLD) review meeting was held and the fit of this synthesis within theoretical models of behaviour change, motivation, human development and health was examined. The findings showed good fit with the biopsychosocial model of health, ${ }^{37}$ which was chosen to facilitate a more comprehensive and meaningful interpretation of the data and reporting of the findings. The descriptive themes were then re-examined and refined. At this point, the research question was introduced to help infer the barriers and facilitators under the three domains of the biopsychosocial model. To enhance the credibility of the findings, the synthesis was conducted by AMK and checked independently by NE.

\section{Additional analysis}

The descriptive study characteristics were examined in relation to the secondary research objectives. Due to insufficient evidence, no further analysis was conducted.

\section{RESULTS}

\section{Study selection}

Five thousand four hundred and forty-nine studies were identified, and after removing duplicates, 2657 titles or/ and abstracts were screened and 51 full-text papers were assessed. Seven authors were contacted for further information. Information was not provided for two studies, which were excluded. Ten studies were included ${ }^{38-47}$ (figure 1).

\section{Study characteristics}

There were 173 participants, mainly middle aged to older, and female. Nine of 10 studies reported qualitative methodologies (table 1).

\section{Appraisal of studies}

All selected studies were of medium or high quality (table 2). The research design and data analysis were not clear or well described in half of the studies and very few studies had clearly identified the relationship between the researcher and participants. Credibility, transferability and confirmability were met by almost all studies, although dependability only by two.

\section{Synthesis of results}

Barriers and facilitators are presented under the three conceptual domains, that is, physical health, intrapersonal factors and social-environmental factors. Barriers and facilitators that appeared in at least three studies are reported to keep a balance between richness and applicability of the findings (table 3; see online supplementary file 3 for supporting references). When comparing exercise and PA focused studies, the themes were similar in context and equally represented in most cases. Where there are differences, these are reported.

\section{Physical health}

\section{Barriers}

Physical barriers and limitations. Pain is aversive, stressful and inherent to living with OA. ${ }^{38-46}$ It was mentioned as part of daily experience ${ }^{445}$ or in relation to particular types of activities. ${ }^{39-41} 434546$ Along with fatigue and stiffness, ${ }^{43-45}$ these symptoms hindered the ability to engage in PA. There was a vicious cycle between symptoms and lack of exercise. ${ }^{40}{ }^{41}$ At an advanced stage of OA, PA was inhibited. ${ }^{41} \mathrm{OA}$ symptoms were aggravated by obesity and made PA more difficult. ${ }^{3841} 44$ Participants also discussed their sense of limited physical capacities and that one's body cannot manage PA requirements, resulting in loss of previous activity patterns. ${ }^{41-45}$ For example, some talked about the need to choose between activities because of limited energy. ${ }^{43}$ Old age and lack of physical fitness were also reported as perceived PA barriers. ${ }^{41} 44$

\section{Facilitators: PA for mobility, symptom relief and health}

Among those who held a physically active lifestyle maintaining or regaining their mobility was a strong motive for PA. ${ }^{38} 40444547$ In most cases, the aim was to keep functioning, ${ }^{38} 414346$ in some it was so specific as to prevent joint surgery. ${ }^{40} 47$ Pain relief is another strong motive for being physically active and active individuals were more likely those who had experienced pain reduction. ${ }^{3840444547}$ A few informants presented a 'no pain, no maintenance' pattern, where pain cessation was followed by dropping exercise. ${ }^{38}$ Improvements in other symptoms, such as stiffness and joint stability, were sufficient reasons for being active, even when pain remained. ${ }^{384}$ Maintaining good general health and physical condition were also reasons for being physically active. ${ }^{40} 41434446$ This facilitator was closely linked to a positive, beneficial PA experience and subsequent positive attitude towards $\mathrm{PA}$, which is a crucial facilitator discussed below.

\section{Intrapersonal/psychological factors}

Experience and beliefs about exercise

Facilitators. Exercise as beneficial. Experiencing benefits from exercise participation, which in most of the studies was related to engagement in an exercise intervention, helped shaping positive beliefs and motivated individuals towards continuing exercise. ${ }^{38-41} 444647$ A sense of 


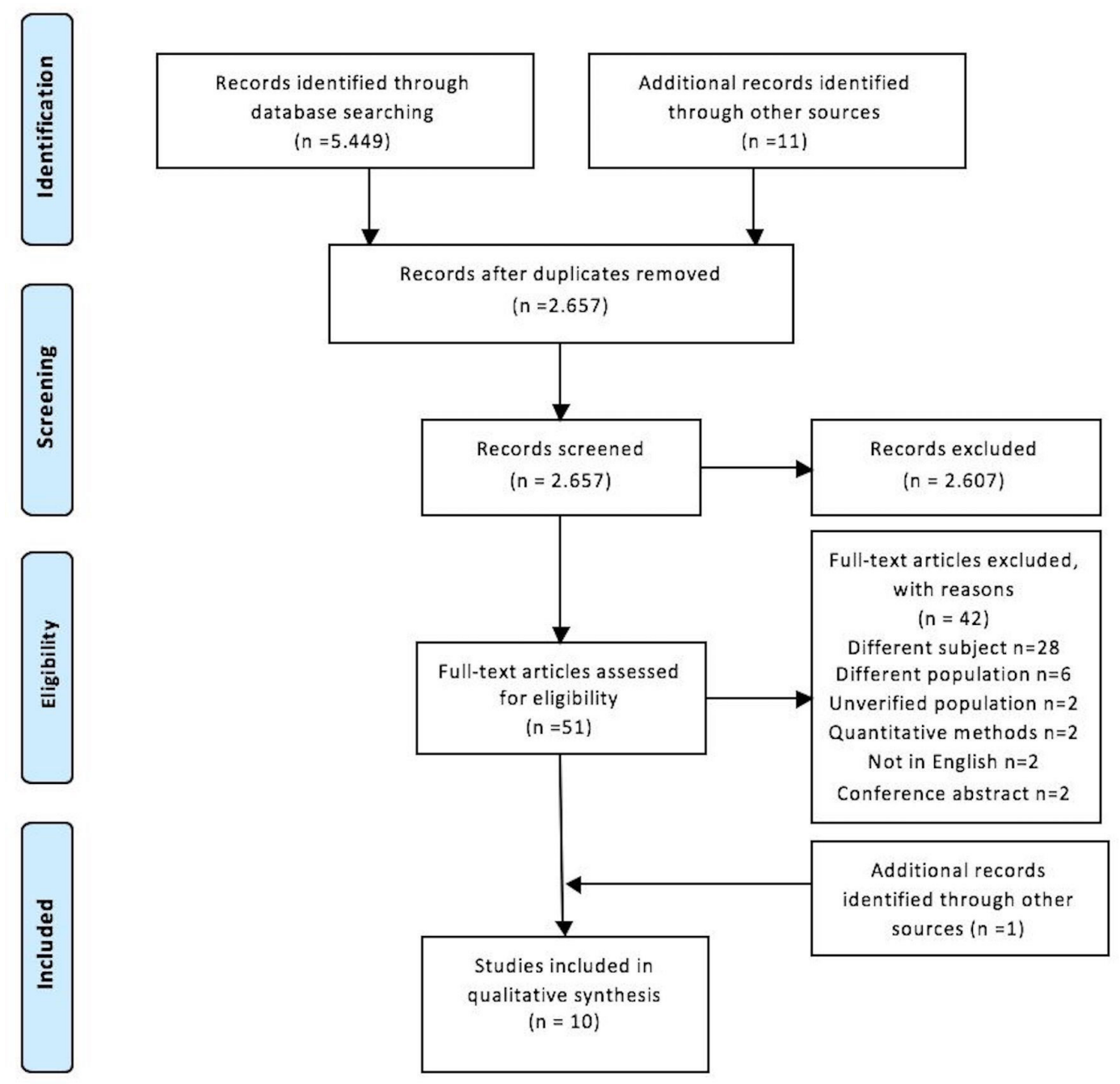

From: Moher D, Liberati A, Tetzlaff J, Altman DG, The PRISMA Group (2009). Preferred Reporting Mems for Systematic Reviews and MetaAnalyses: The PRISMA Statement. PLoS Med 6(7): e1000097. doi:10.1371 fiouchahponed1000097

For more information, visit www.prisma-statement.org.

Figure 1 Study selection Preferred Reporting Items for Systematic Reviews and Meta-Analyses flow diagram.

psychosomatic well-being was an important component of this theme. ${ }^{39-41446}$ Improvement in coping with $\mathrm{OA}^{46}$ and sleep ${ }^{44}$ were mentioned.

Knowledge about exercise in OA. Accurate knowledge of the importance of exercise in OA, acquired through healthcare, physiotherapy and exercise interventions, was an important facilitator. ${ }^{40-46}$ It led to awareness regarding exercise benefits and helped in making correct interpretations of exercise experiences.

Both the above themes emerged from exercise-focused studies only.

Barriers. PA as non-effective, harmful or of doubtful effectiveness. The belief that PA does not help or might further deteriorate their condition hindered people from being active. ${ }^{3840} 41434546$ Experiencing activity-related pain in the joint, for example, was often interpreted as PA exacerbating $\mathrm{OA}$, which stemmed from the understanding of OA as a 'wear and tear' condition. ${ }^{41}{ }^{43}$ Not experiencing the anticipated beneficial effects during exercise interventions was a reason for distrust in PA as an effective means of treatment. ${ }^{38404146}$ Also, early negative experiences with sports resulted in exercise avoidance. ${ }^{44}$

$O A$ beliefs. Beliefs that nothing can be done regarding the condition ${ }^{41446}$ and that overuse was the cause of $\mathrm{OA}^{384143}$ were linked to less inclination towards being physically active. In one study, the relationship between PA and OA was discussed as bidirectional. ${ }^{43}$ These beliefs were mostly reported in exercise-focused studies (four exercise studies with one PA-focused study also revealing such beliefs).

Daily activities as $P A$. This theme revolved around beliefs about non-leisure PA. ${ }^{41} 4346$ However, there were no consistent patterns across studies to be clearly classified as barriers or facilitators. For example, non-leisure activities 


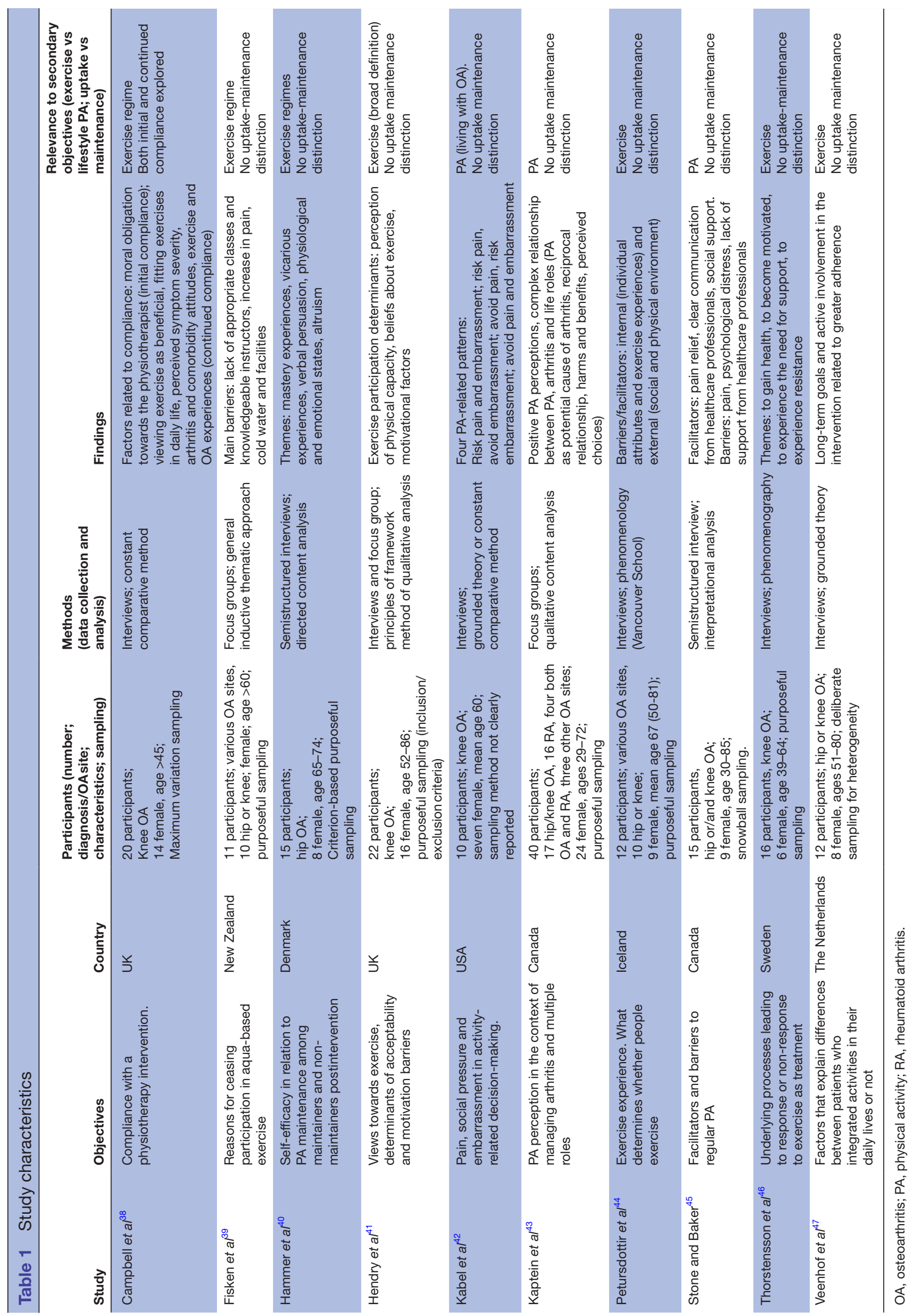




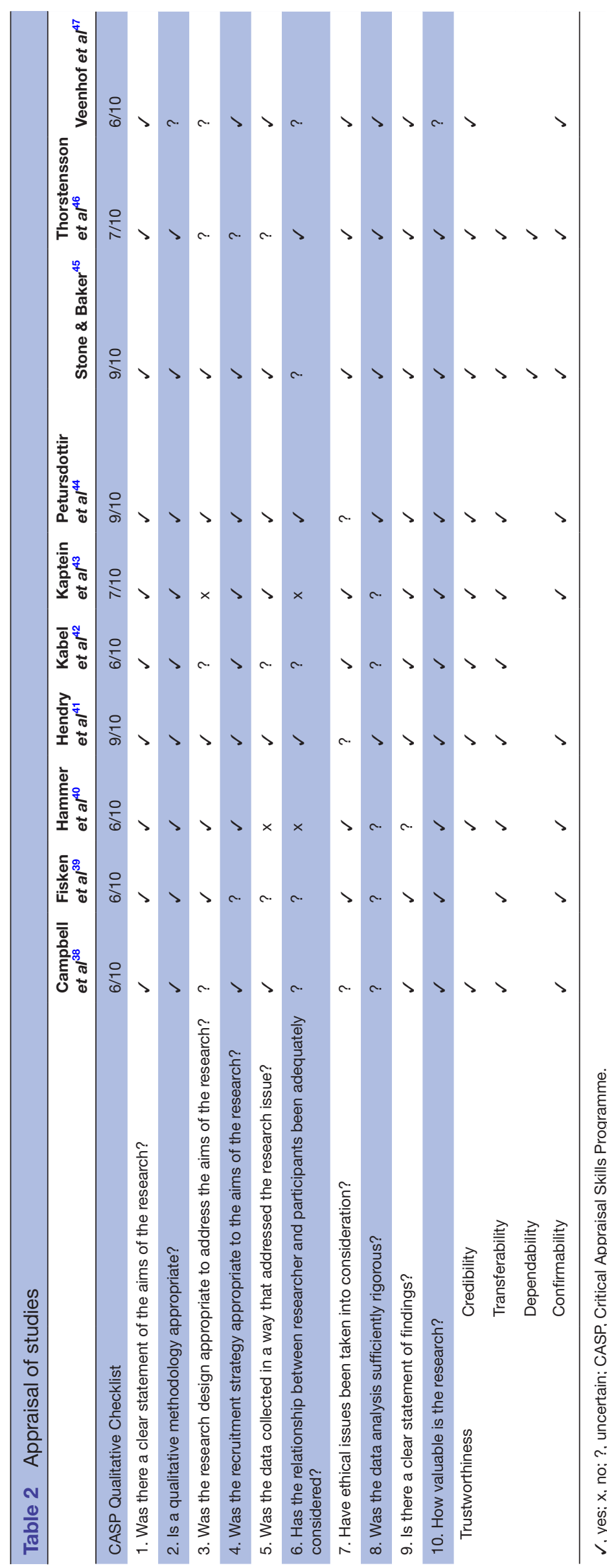

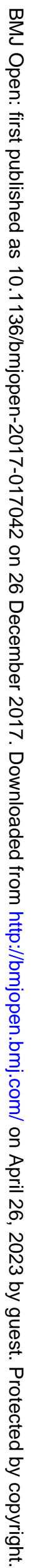


Table 3 Barriers and facilitators: themes, subthemes and number of supporting references

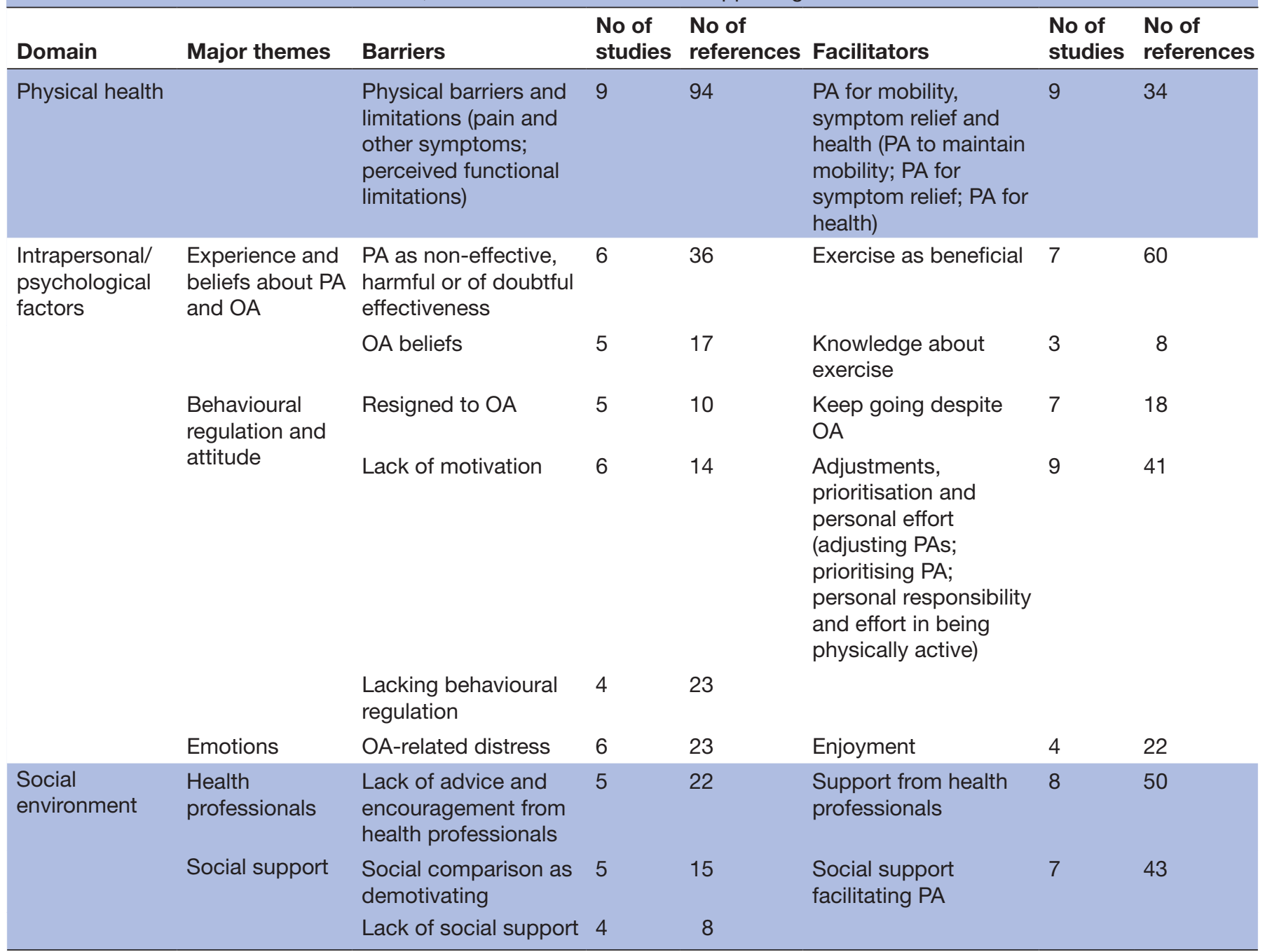

OA, osteoarthritis; PA, physical activity.

were viewed as a sufficient amount of PA by some ${ }^{41} 4446$ and as insufficient by others. ${ }^{41}$

\section{Behavioural regulation and attitude}

Facilitators. Keep going despite OA. Authors' interpretations related to this concept varied, for example, determination to take control of arthritis, ${ }^{41}$ perseverance, ${ }^{46}$ personality traits of adaptability and initiative, ${ }^{44}$ belief that there are 'things patients can do' about their $\mathrm{OA}^{38}$ and motivation towards long-term goals. ${ }^{47}$ The importance of keeping a positive attitude was also discussed. ${ }^{43}$ In two studies, the relevant participant quotes were presented under the themes 'risking embarrassment ${ }^{\text {'42 }}$ and 'bidirectional impact between PA and arthritis'. 43

Adjustments, prioritisation and personal effort. Physically active individuals described how they were making short or long-term modifications to their $\mathrm{PA},{ }^{39-44}$ such as finding a type of exercise that was suitable for their physical abilities, ${ }^{39-41} 44$ adjusting PA intensity to their current condition, ${ }^{40} 4244$ even changing their job. ${ }^{43}$ This task of continuously adjusting PAs was quite demanding. ${ }^{44}$ Prioritising PA and fitting it into a routine was mentioned by a number of physically active participants and reflected the importance they assigned to PA. ${ }^{3841} 4647$ Active participants also acknowledged they were the main agents in managing their condition and they were consciously making efforts to stay active. 38414346

Barriers. Lack of motivation. Participants in different studies referred to a lack of motivation or goal, laziness and boredom towards exercise. ${ }^{38} 4041444647$ These type of barriers were reported in the exercise-focused studies only and were not further explored.

Lacking behavioural regulation. In the face of the demands of other life roles and a busy schedule, especially family related, inactive participants were not prioritising PAs. ${ }^{38} 414346$ In two studies, informants referred to not finding a PA suitable for their current condition. ${ }^{39} 41$ In one study, low self-regulation was the reason given for not exercising regularly. ${ }^{41}$

Resigned to OA. In half of the studies, informants expressed a resigned attitude towards making an effort to be active. ${ }^{3841} 44-46$ Reflecting fatalistic beliefs about OA and feelings of helplessness, this attitude was linked to attenuated motivation for being physically active. 


\section{Emotions}

Facilitators. Enjoyment. Enjoying exercise in general or a particular type of exercise facilitated its continuation. ${ }^{39} 414$ This facilitator of engagement emerged in the exercise-focused studies only.

Barriers. OA-related distress. Living with OA means adjusting to a reality of decreased physical functioning and in several cases, participants talked about this experience of giving up activities, being unable to meet life roles and daily demands as distressing or embarrassing. ${ }^{38} 4042-45$ Mental stress, ${ }^{40}$ extreme unhappiness and paralysing fatigue, ${ }^{44}$ feeling broken and mentally depressed, ${ }^{45}$ weakness ${ }^{43}$ were used.

\section{Social environment}

Health professionals

Facilitators. Support from health professionals. Physiotherapists exerted great influence on the patients' PA/exercise habits. ${ }^{38} 4041444547$ Providing instructions, education, encouragement and rapport with the patient were means of facilitating exercise. Advice and prescription by doctors was another facilitator. ${ }^{41} 44$ Supervision during exercise was valued. ${ }^{38-41} 4647$ Good supervision gave participants the reassurance that what they were doing was appropriate and good for their body, ${ }^{41}$ which they needed, ${ }^{45}$ and motivated them to exercise. ${ }^{38} 404647$ Support from health professionals was reported in four exercise studies and one PA focused.

Barriers. Lack of support from health professionals. Ambiguous, no or conflicting information from doctors regarding PA was a barrier. ${ }^{38414445}$ In one study, the instructor not having specialised $\mathrm{OA}$ training was the reason that lead participants to discontinue their exercise. ${ }^{39}$

\section{Social support}

Facilitators. Social support facilitating PA. Social support as a facilitator was mainly discussed in the context of exercising in a group, as well as support from family and friends. Feeling comfortable and motivated, even inspired when exercising with people of similar physical abilities and age emerged as an advantage of PA programmes. ${ }^{38-41} 4345$ This was of particular importance when someone was first introduced to PA. ${ }^{40}$ Opportunities to socialise were also an advantage of group PA. ${ }^{39} 41$ In addition, psychological and instrumental support from family and friends emerged as an asset of physically active participants, taking the form of active encouragement, expression of interest and understanding, an exercise buddy or role model. ${ }^{40-45}$ Community-based support was mentioned as PA promoting. ${ }^{45}$ This theme stemmed equally from exercise and PA-centred studies, although the focus of the former was on group exercise and the latter on important others' support for an active lifestyle.

Barriers. Social comparison as demotivating. Although this concept did not explicitly appear as an authors' interpretation, it emerged from informants' quotes. Being unable to keep up with others when participating in PA was a PA barrier as it provoked feelings of embarrassment and distress. ${ }^{38-42}$ This barrier was reported in four exercise studies and one PA focused.

Lack of social support. The lack of social support from peers and family as a barrier was discussed in relation to lack of understanding and encouragement from the person's family and social ${ }^{384344}$ and work environment. ${ }^{43}$

\section{Physical environment}

Barriers. The cost of exercise classes, ${ }^{39} 4344$ limited accessibility $^{446}$ and lack of availability of appropriate modes, ${ }^{44} 45$ as well as cold weather and issues regarding safety ${ }^{39}$ were the reported environmental barriers to PA.

\section{DISCUSSION}

This $\mathrm{SR}$ is the first to synthesise the existing qualitative research on barriers and facilitators to PA in knee and hip OA. Pain and physical limitations, absence of positive PA experiences and beliefs, resigned attitude and distress due to OA, lack of behavioural regulation, lack of support from health professionals and negative social comparisons when exercising in a group were important PA barriers. Symptom relief and mobility, positive exercise experiences and beliefs, knowledge, enjoying exercise, a 'keep going' attitude, adjusting and prioritising PA and having professional and social support were important PA facilitators. Overall, the findings are consistent with known PA correlates in exercise psychology, ${ }^{48}$ theories of behavioural change ${ }^{49}$ and results emanating from existing SRs in general (ie, non-OA specific) populations that share common characteristics with OA patients. ${ }^{50-52}$ Present findings also outline a unique profile of PA barriers and facilitators in lower limb OA.

Factors related to physical health, specifically pain and physical function, were the most consistently reported. This indicates that OA has a central role and impact in people's lives and experiences, which is in line with previous qualitative findings that pain discussions by people with OA differ in frequency and quality in comparison to healthy individuals. ${ }^{53}$ Importantly, physical barriers are reported both by active and inactive people. Therefore, physical barriers alone cannot explain PA behaviour with the exception of patients at very advanced stages of $\mathrm{OA}^{54}$ Intrapersonal and social variables are crucial in PA behaviours reported earlier. $^{52}$

The identified barriers and facilitators are not standalone and independent entities but manifest a complex interplay. Personal experience, knowledge and beliefs about PA, exercise and OA were interwoven concepts and formed the basis of PA behaviour. Experiencing benefits from participation in an exercise programme, which was the case in most of the included studies, shapes a positive attitude towards PA. ${ }^{50} 51$ 55-57 Accurate knowledge regarding $\mathrm{PA}$, exercise and OA bolstered a positive interpretation of and predisposition towards PA experience. Viewing pain as manageable versus inevitable elicited different behaviors ${ }^{58} 59$ and, not surprisingly, patient education is a core component of healthcare and 
OA management. ${ }^{60}$ Support from health professionals becomes crucial as they can provide rationale and motivation for $\mathrm{PA}^{55}$ and shape the patients' health experience. ${ }^{53}$ The above factors and available social support are not independent from, but influence motivation, attitude and behavioural regulation.

Most of the PA barriers and facilitators emerged under the psychological/intrapersonal domain and were mostly OA related. The data analysis allowed for new insights into the original studies, such as the emerging theme of OA-related distress and two distinct patterns in attitude, beliefs, motivation and behavioural regulation-one facilitating and the other hindering PA. Pain and its multifaceted impact is a source of distress in OA. ${ }^{24}$ In turn, anxiety and depressive symptoms, which are more prevalent in people with arthritis, ${ }^{61}$ are predictors of poorer function $^{6263}$ and pain. ${ }^{2864-66}$ Still cognitive processes underlying the distinct patterns are missing, for example, what distinguishes those who, for a given level of structural disease-severity and OA-related pain, exhibit a positive attitude and behavioural regulation from those who are resigned, cope ineffectively with OA stress and lack self-regulation? Explanations involving distinguishing processes and participant characteristics might lie in theoretical frameworks of behaviour change and health, which are absent in the included studies, with one exception. ${ }^{40}$ For example, self-efficacy, self-determination and need satisfaction are precursors of behaviour in theories which have been applied to predicting and promoting $\mathrm{PA},{ }^{6768}$ whereas sense of control is a common concept in the stress and coping literature. ${ }^{69}$ Future research should make use of theoretical knowledge and approaches to enable targeted and more effective research and interventions. ${ }^{70}$

All the findings reported were grounded in the three studies that scored 'high' at both sets of quality criteria, ${ }^{41445}$ along with the seven medium quality studies, which confirms their trustworthiness. However, aspects of methodology were poorly reported or explored in the selected studies, particularly those of medium quality. A consideration of the researcher-participant relationship and employing an external auditor for the decision trail (dependability) should be used to increase confidence in the findings.

The SR findings hold implications for clinical practice. All healthcare professionals who manage people with lower limb OA have a key role in facilitating PA through their advice, attitude towards $\mathrm{OA}$ and decision to seek multidisciplinary input for example, from physiotherapy. Even without directed advice to increase PA, health and condition-related advice and a supportive stance from healthcare professionals can influence decisions related to PA engagement. ${ }^{71}$ In the absence of education, people with $\mathrm{OA}$ tend to draw from lay and often fatalistic beliefs of PA and exercise in OA. An individual assessment of the experienced impact of pain and disability, personal attitudes and circumstances, educating about the role of PA in $\mathrm{OA}$ management, offering feasible yet specific PA prescription and encouragement can have an impact on the persons' PA and exercise behaviour. Pain and stress-related coping strategies, guidance through exercise prescription and effective communication are the main components of established arthritis self-management programmes. ${ }^{72}$ Increasing the time designated to each patient within the healthcare system could allow for such practices to take place. Counselling referral and online educational tools could also affect PA behaviour.

Based on the available qualitative evidence, it was not possible to adequately explore the secondary SR questions, an issue which has been previously reported.$^{5273}$ Only three studies focused on lifestyle PA, which is surprising considering the paradigm shift in the health literature from exercise promotion to a combination of PA promotion and sedentary time reduction. ${ }^{74}$ Also, only one study made the distinction between PA uptake and maintenance, despite the recognition that these two stages entail different determinants. ${ }^{67} 75$ In the case of people living with OA, the factors and processes leading to uptake and maintenance of overall PA need to be further explored and understood.

This SR has applied rigorous methods and provides an in-depth and meaningful understanding of the phenomenon of interest based on the accumulated existing qualitative evidence, thus moving one step forward from existing SRs. ${ }^{21}{ }^{22}$ Gaps in the existing literature were also identified. With regards to data synthesis, coding participants' quotes and authors' interpretations separately allowed aspects of the phenomenon not captured by the original studies to come to light. During data synthesis, peer review by a multidisciplinary team took place to enhance credibility. The main reviewer's background is clinical psychology, which might be reflected in the emphasis on the 'psychological' component of PA barriers and facilitators.

There are certain limitations to this study. The majority of the included studies were exercise focused, therefore might not accurately or fully represent barriers and facilitators to lifestyle PA (of which engaging in structured exercise programme is type or form). Due to resource limitations, studies not written in English were excluded. Two relevant studies were also excluded because they were in a conference abstract form and additional data were not available. ${ }^{77} 78$ Lastly, due to the nature of the evidence, directions of the relationships and interactions among the identified factors cannot be drawn.

In summary, there is a complex interplay among the physical, intrapersonal, psychological and socio environmental barriers and facilitators of exercise and PA that bears similarities with other chronic diseases, but also includes characteristics specific to OA. Personal experiences, beliefs, attitudes and emotions, as well as the social environment, that is, healthcare and social support, are dynamic factors shaping PA behaviour. Considering that OA becomes more prevalent with age, it is important and challenging to make sustained lifestyle changes that will have a positive impact on an individual as well as at a healthcare system level. With the aim of identifying effective practices to help people with OA become more active, future research should involve behavioural intervention studies to address the factors identified above. 


\section{AMENDMENTS TO THE PROTOCOL}

Confidence in the synthesised findings was not used due to ambiguities in the suggested process $\left(\mathrm{ConQual}^{79}\right)$, that is, regarding transparency and satisfactory justification of the assessment outcome. However, the studies-sources of each finding were checked. The three studies scoring 'high' quality at both sets of criteria informed all themes, along with the medium quality studies.

Kappa statistic was not measured. The two researchers run the searches independently for all databases following the Medline search strategy. Because of differences in operators and options at different search engines, the number of studies differed at the stages preceding study selection. Each reviewer's full text selection stage was updated by the other researcher's findings. At this stage agreement was met for all included studies.

\section{Author affiliations}

${ }^{1}$ School of Sport, Exercise and Rehabilitation Sciences, College of Life and Environmental Sciences, University of Birmingham, Birmingham, UK

${ }^{2}$ MRC-Arthritis Research UK Centre for Musculoskeletal Ageing Research, University of Birmingham, Birmingham, UK

${ }^{3}$ Centre of Precision Rehabilitation for Spinal Pain (CPR Spine), University of Birmingham, Birmingham, UK

${ }^{4}$ Nursing, Institute of Clinical Sciences, Medical School, University of Birmingham, Birmingham, UK

${ }^{5}$ Department of Health Rehabilitation Sciences, King Saud University, Riyadh, Saudi Arabia

${ }^{6}$ Department of Rheumatology, Dudley Group NHS Foundation Trust, Dudley, UK ${ }^{7}$ Academic Rheumatology Unit, School of Medicine, University of Nottingham, Nottingham, UK

${ }^{8}$ School of Nursing, University of Ottawa, Ottawa, Canada

Contributors Study concept and design: JLD, AR, AMK, RK, AbA, NE. Searches: AMK, AsA. Study appraisal: AMK, NE. Data analysis: AMK, checked by NE. Data interpretation: AMK, checked by JLD, AR, NE. Manuscript draft: AMK. Manuscript review and input: JLD, AR, AbA, NE, RK. All authors provided feedback and approved the final draft.

Funding This review comprises part of the research requirements of a $\mathrm{PhD}$ to be completed by AMK, funded by the MRC-Arthritis Research UK Centre for Musculoskeletal Ageing Research.

Competing interests None declared.

Provenance and peer review Not commissioned; externally peer reviewed.

Data sharing statement Qualitative synthesis level electronic data (NVivo V.11) are available upon request from the corresponding author.

Open Access This is an Open Access article distributed in accordance with the terms of the Creative Commons Attribution (CC BY 4.0) license, which permits others to distribute, remix, adapt and build upon this work, for commercial use, provided the original work is properly cited. See: http://creativecommons.org/ licenses/by/4.0/

(C) Article author(s) (or their employer(s) unless otherwise stated in the text of the article) 2017. All rights reserved. No commercial use is permitted unless otherwise expressly granted.

\section{REFERENCES}

1. NICE. Osteoarthritis care and management in adults. NICE clinical guideline 2014;177.

2. McAlindon TE, Bannuru RR, Sullivan MC, et al. OARSI guidelines for the non-surgical management of knee osteoarthritis. Osteoarthritis Cartilage 2014;22:363-88.

3. Caspersen CJ, Powell KE, Christenson GM. Physical activity, exercise, and physical fitness: definitions and distinctions for healthrelated research. Public Health Rep 1985;100:126-31.
4. Fransen M, McConnell S, Harmer AR, et al. Exercise for osteoarthritis of the knee: a Cochrane systematic review. Br J Sports Med 2015;49:1554-7.

5. Fransen M, McConnell S, Hernandez-Molina G, et al. Exercise for osteoarthritis of the hip. Cochrane Database Syst Rev 2014;2014.

6. Anwer S, Alghadir A, Brismée JM. Effect of home exercise program in patients with knee osteoarthritis: a systematic review and metaanalysis. J Geriatr Phys Ther 2016;39:38-48.

7. Juhl C, Christensen R, Roos EM, et al. Impact of exercise type and dose on pain and disability in knee osteoarthritis: a systematic review and meta-regression analysis of randomized controlled trials. Arthritis Rheumatol 2014;66:622-36.

8. Boesen MI, Boesen A, Koenig MJ, et al. Ultrasonographic investigation of the Achilles tendon in elite badminton players using color Doppler. Am J Sports Med 2006;34:2013-21.

9. Uthman OA, van der Windt DA, Jordan JL, et al. Exercise for lower limb osteoarthritis: systematic review incorporating trial sequential analysis and network meta-analysis. [Reprint of BMJ. 2013;347:f5555; PMID: 24055922]. Br J Sports Med 2014;48:1579.

10. Pisters MF, Veenhof C, van Meeteren NL, et al. Long-term effectiveness of exercise therapy in patients with osteoarthritis of the hip or knee: a systematic review. Arthritis Rheum 2007;57:1245-53.

11. Pisters MF, Veenhof $\mathrm{C}$, de Bakker $\mathrm{DH}$, et al. Behavioural graded activity results in better exercise adherence and more physical activity than usual care in people with osteoarthritis: a clusterrandomised trial. J Physiother 2010;56:41-7.

12. Batsis JA, Germain CM, Vásquez E, et al. Physical activity predicts higher physical function in older adults: the osteoarthritis initiative. $J$ Phys Act Health 2016;13:6-16.

13. Chmelo E, Nicklas B, Davis C, et al. Physical activity and physical function in older adults with knee osteoarthritis. $J$ Phys Act Health 2013;10:777-83.

14. Lin W, Alizai H, Joseph GB, et al. Physical activity in relation to knee cartilage T2 progression measured with $3 \mathrm{~T}$ MRI over a period of 4 years: data from the Osteoarthritis Initiative. Osteoarthritis Cartilage 2013;21:1558-66.

15. Loprinzi PD, Sheffield J, Tyo BM, et al. Accelerometer-determined physical activity, mobility disability, and health. Disabil Health J 2014;7:419-25

16. Austin S, Qu H, Shewchuk RM. Association between adherence to physical activity guidelines and health-related quality of life among individuals with physician-diagnosed arthritis. Qual Life Res 2012;21:1347-57.

17. Austin J, Williams WR, Hutchison S. Multidisciplinary management of elderly patients with chronic heart failure: five year outcome measures in death and survivor groups. Eur $\mathrm{J}$ Cardiovasc Nurs 2009;8:34-9.

18. Herbolsheimer F, Schaap LA, Edwards MH, et al. Physical activity patterns among older adults with and without knee osteoarthritis in six European countries. Arthritis Care Res 2016;68:228-36.

19. Marks R. Knee osteoarthritis and exercise adherence: a review. Curr Aging Sci 2012;5:72-83.

20. Marks R, Allegrante JP. Chronic osteoarthritis and adherence to exercise: a review of the literature. J Aging Phys Act 2005;13:434-60.

21. Stubbs B, Hurley M, Smith T. What are the factors that influence physical activity participation in adults with knee and hip osteoarthritis? A systematic review of physical activity correlates. Clin Rehabil 2015;29:80-94.

22. Veenhof $C$, Huisman PA, Barten JA, et al. Factors associated with physical activity in patients with osteoarthritis of the hip or knee: a systematic review. Osteoarthritis Cartilage 2012;20:6-12.

23. Dobson F, Bennell KL, French SD, et al. Barriers and facilitators to exercise participation in people with hip and/or knee osteoarthritis: synthesis of the literature using behavior change theory. Am J Phys Med Rehabil 2016;95:372-89.

24. Hawker GA, Stewart L, French MR, et al. Understanding the pain experience in hip and knee osteoarthritis--an OARSI/OMERACT initiative. Osteoarthritis Cartilage 2008;16:415-22.

25. Sánchez-Herán Á, Agudo-Carmona D, Ferrer-Peña R, et al. Postural Stability in Osteoarthritis of the Knee and Hip: Analysis of Association With Pain Catastrophizing and Fear-Avoidance Beliefs. Pm R 2016;8:618-28.

26. Holla JF, van der Leeden M, Knol DL, et al. Predictors and outcome of pain-related avoidance of activities in persons with early symptomatic knee osteoarthritis: a five-year followup study. Arthritis Care Res 2015;67:48-57.

27. Holla JF, Sanchez-Ramirez DC, van der Leeden M, et al. The avoidance model in knee and hip osteoarthritis: a systematic review of the evidence. J Behav Med 2014;37:1226-41. 
28. Somers TJ, Keefe FJ, Godiwala N, et al. Psychosocial factors and the pain experience of osteoarthritis patients: new findings and new directions. Curr Opin Rheumatol 2009;21:501-6.

29. Wideman TH, Finan PH, Edwards RR, et al. Increased sensitivity to physical activity among individuals with knee osteoarthritis: relation to pain outcomes, psychological factors, and responses to quantitative sensory testing. Pain 2014;155:703-11.

30. Arksey H, O'Malley L. Scoping studies: towards a methodological framework. Int J Soc Res Methodol 2005;8:19-32.

31. Kanavaki AM, Rushton A, Klocke R, et al. Barriers and facilitators to physical activity in people with hip or knee osteoarthritis: protocol for a systematic review of qualitative evidence. BMJ Open 2016;6:e012049.

32. Altman RD. Criteria for classification of clinical osteoarthritis. $J$ Rheumatol Suppl 1991;27:10-12.

33. H K. et a/Critical appraisal of qualitative research. In: Noyes JBA, Hannes K, Harden A, ; eds. Supplementary Guidance for Inclusion of Qualitative Research in Cochrane Systematic Reviews of Interventions Version 1 (updated August 2011): Cochrane Collaboration Qualitative Methods Group, 2011.

34. Critical Appraisal Skills Programme (CASP). CASP Checklists. Oxford: CASP, 2014 http://wwwcasp-uknet/ - !checklists/cb36

35. Creswell JW. Qualitative Inquiry and Research Design. Thousand Oaks, California: SAGE Publications Inc, 2007.

36. Barnett-Page E, Thomas J. Methods for the synthesis of qualitative research: a critical review. BMC Med Res Methodol 2009;9:59.

37. Engel GL. The clinical application of the biopsychosocial model. Am J Psychiatry 1980;137:535-44.

38. Campbell R, Evans M, Tucker M, et al. Why don't patients do their exercises? Understanding non-compliance with physiotherapy in patients with osteoarthritis of the knee. J Epidemiol Community Health 2001;55:132-8.

39. Fisken AL, Waters DL, Hing WA, et al. Perceptions towards aquabased exercise among older adults with osteoarthritis who have discontinued participation in this exercise mode. Australas $J$ Ageing 2016;35.

40. Hammer NM, Bieler T, Beyer N, et al. The impact of self-efficacy on physical activity maintenance in patients with hip osteoarthritis - a mixed methods study. Disability and rehabilitation 2015;1-14.

41. Hendry M, Williams NH, Markland D, et al. Why should we exercise when our knees hurt? A qualitative study of primary care patients with osteoarthritis of the knee. Fam Pract 2006;23:558-67.

42. Kabel A, Dannecker EA, Shaffer VA, et al. Osteoarthritis and social embarrassment: Risk, Pain, And avoidance. SAGE Open 2014;4.

43. Kaptein SA, Backman CL, Badley EM, et al. Choosing where to put your energy: a qualitative analysis of the role of physical activity in the lives of working adults with arthritis. Arthritis Care Res 2013:65:1070-6.

44. Petursdottir U, Arnadottir SA, Halldorsdottir S. Facilitators and barriers to exercising among people with osteoarthritis: a phenomenological study. Phys Ther 2010;90:1014-25.

45. Stone RC, Baker J. Painful choices: a qualitative exploration of facilitators and barriers to active lifestyles among adults with osteoarthritis. J Appl Gerontol 2015.

46. Thorstensson CA, Roos EM, Petersson IF, et al. How do middleaged patients conceive exercise as a form of treatment for knee osteoarthritis? Disabil Rehabil 2006;28:51-9.

47. Veenhof $\mathrm{C}$, van Hasselt TJ, Koke AJ, et al. Active involvement and long-term goals influence long-term adherence to behavioural graded activity in patients with osteoarthritis: a qualitative study. Aust $J$ Physiother 2006:52:273-8.

48. Biddle SJH, Mutrie N. Psychology of physical activity. Determinants, well-being \& interventions. 2nd ed. New York: Routledge, 2008:33-160

49. Kwasnicka D, Dombrowski SU, White M, et al. Theoretical explanations for maintenance of behaviour change: a systematic review of behaviour theories. Health Psychol Rev 2016;10:277-96.

50. Franco MR, Tong A, Howard K, et al. Older people's perspectives on participation in physical activity: a systematic review and thematic synthesis of qualitative literature. Br J Sports Med 2015;49:1268-76.

51. Morgan F, Battersby A, Weightman AL, et al. Adherence to exercise referral schemes by participants - what do providers and commissioners need to know? A systematic review of barriers and facilitators. BMC Public Health 2016;16:227.

52. Rhodes RE, Quinlan A. Predictors of physical activity change among adults using observational designs. Sports Med 2015;45:423-41.

53. Gignac MA, Davis AM, Hawker G, et al. "What do you expect? You're just getting older": a comparison of perceived osteoarthritis-related and aging-related health experiences in middle- and older-age adults. Arthritis Rheum 2006;55:905-12.
54. Liu SH, Driban JB, Eaton CB, et al. Objectively measured physical activity and symptoms change in knee osteoarthritis. Am J Med 2016;129:497-505

55. Horne M, Tierney S. What are the barriers and facilitators to exercise and physical activity uptake and adherence among South Asian older adults: a systematic review of qualitative studies. Prev Med 2012:55:276-84.

56. Wilcox S, Der Ananian C, Abbott J, et al. Perceived exercise barriers, enablers, and benefits among exercising and nonexercising adults with arthritis: results from a qualitative study. Arthritis Rheum 2006;55:616-27.

57. Peeters GM, Brown WJ, Burton NW. Psychosocial factors associated with increased physical activity in insufficiently active adults with arthritis. J Sci Med Sport 2015;18:558-64.

58. Main CJ, Williams AC. Musculoskeletal pain. BMJ 2002;325:534-7.

59. Ross MM, Carswell A, Hing M, et al. Seniors' decision making about pain management. J Adv Nurs 2001;35:442-51.

60. Larmer PJ, Reay ND, Aubert ER, et al. Systematic review of guidelines for the physical management of osteoarthritis. Arch Phys Med Rehabil 2014;95:375-89.

61. He Y, Zhang M, Lin EH, et al. Mental disorders among persons with arthritis: results from the World Mental Health Surveys. Psychol Med 2008;38:1639-50.

62. Dekker J, Boot B, van der Woude LH, et al. Pain and disability in osteoarthritis: a review of biobehavioral mechanisms. J Behav Med 1992;15:189-214.

63. Sharma L, Cahue S, Song J, et al. Physical functioning over three years in knee osteoarthritis: role of psychosocial, local mechanical, and neuromuscular factors. Arthritis Rheum 2003;48:3359-70.

64. Edwards RR, Dworkin RH, Sullivan MD, et al. The role of psychosocial processes in the development and maintenance of chronic pain. J Pain 2016;17(9 Suppl):T70-T92.

65. de Rooij M, van der Leeden M, Avezaat E, et al. Development of comorbidity-adapted exercise protocols for patients with knee osteoarthritis. Clin Interv Aging 2014;9:829-42.

66. Volders S, Boddez Y, De Peuter S, et al. Avoidance behavior in chronic pain research: a cold case revisited. Behav Res Ther 2015;64:31-7.

67. Deci EL, Ryan RM. Self-determination theory: A macrotheory of human motivation, development, and health. Canadian Psychology/ Psychologie canadienne 2008:49:182-5.

68. Bandura A. Self-efficacy: toward a unifying theory of behavioral change. Psychol Rev 1977;84:191-215.

69. Jensen MP, Turner JA, Romano JM, et al. Coping with chronic pain: a critical review of the literature. Pain 1991;47:249-83.

70. Michie S. Designing and implementing behaviour change interventions to improve population health. $J$ Health Serv Res Policy 2008;13(Suppl 3):64-9.

71. Horne M, Skelton D, Speed S, et al. The influence of primary health care professionals in encouraging exercise and physical activity uptake among White and South Asian older adults: experiences of young older adults. Patient Educ Couns 2010;78:97-103.

72. Lorig KR, Mazonson PD, Holman HR. Evidence suggesting that health education for self-management in patients with chronic arthritis has sustained health benefits while reducing health care costs. Arthritis Rheum 1993;36:439-46.

73. Koeneman MA, Verheijden MW, Chinapaw MJ, et al. Determinants of physical activity and exercise in healthy older adults: a systematic review. Int J Behav Nutr Phys Act 2011;8:142.

74. Katzmarzyk PT. Physical activity, sedentary behavior, and health: paradigm paralysis or paradigm shift? Diabetes 2010;59:2717-25.

75. Prochaska JO, DiClemente CC. Stages and processes of self-change of smoking: toward an integrative model of change. J Consult Clin Psychol 1983;51:390-5.

76. Kinnafick FE, Thøgersen-Ntoumani C, Duda JL. Physical activity adoption to adherence, lapse, and dropout: a self-determination theory perspective. Qual Health Res 2014;24:706-18.

77. C Eb G, Levyckyj C, Chabaud A, et al. Facilitators and Barriers in physical activity engagement for knee osteoarthritis patients. 30th Annual Congress of the French Society of Physical and Rehabilitation Medicine. Ann Phys Rehabil Med 2015.

78. Jinks C, Moore A, Holden MA, et al. Uptake and maintenance of physiotherapy-led exercise and general physical activity in older adults with knee pain: The beep longitudinal qualitative study. Rheumatology 2015;54:36.

79. Munn Z, Porritt K, Lockwood C, et al. Establishing confidence in the output of qualitative research synthesis: the ConQual approach. BMC Med Res Methodol 2014;14:1-7. 